\title{
THE EFFECTIVENESS OF CORTICOSTEROID IN BRONCHIOLITIS BY RESPIRATORY SYNCYTIAL VIRUS AND RHINOVIRUS
}

K.H. Kim, S.Y. Kim, M.S. Kim, Y.H. Chun, S.H. Kim

The Catholic University of Korea- Incheon St. Mary's Hospital, Pediatrics, Incheon, Republic of Korea..

\section{Background}

Bronchiolitis is a common lower respiratory tract illness caused by viral infection in children 2 years of age and younger.

Recent practice guidelines recommend limiting use of bronchodilators, corticosteroids, antibiotics, and diagnostic testing for patients with bronchiolitis.

Wheezing episodes induced by various viral infection are diagnosed as bronchiolitis. Although respiratory syncytial virus (RSV) is the major cause of bronchiolitis, other agents cause wheezing episode in young children.

We sought to determine whether corticosteroids are efficacious in treating hospitalized children with wheezing according to causative agents of RSV or rhinovirus.

\section{NICE guideline (June 2015)}

Do not use any of the following to treat bronchiolitis in children: antibiotics, salbutamol and systemic or inhaled corticosteroids etc.

\section{American Academy of Pediatrics (2016)}

Clinicians should not administer systemic corticosteroids to infants with a diagnosis of bronchiolitis in any setting (Evidence Quality: A; Recommendation Strength: Strong Recommendation)

\section{Methods}

This study included children under the 24 months old with acute bronchiolitis or respiratory infection presenting wheezing from June 2016 to Dec 2017.

The infectious viruses were analyzed by multiplex real time PCR.

409 children infected by RSV or rhinovirus were analyzed retrospectively.

Outcome measures was the length of hospital stay (LOS). LOS was compared by virus and by use of steroid.

T-test was applied to compare mean difference among independent groups for the variables length of hospital stay (in days). $\mathrm{P}$ < 0.05 was considered significant.

\begin{tabular}{|c|c|c|c|}
\hline $\begin{array}{c}\text { June, 2016 } \\
\text { Dec, 2017 }\end{array}$ & & M & F \\
\hline RSV A & 142 & 76 & 66 \\
\hline RSV B & 116 & 73 & 43 \\
\hline Rhinovirus & 151 & 84 & 67 \\
\hline
\end{tabular}

Results

Cases of rhinovirus, RSV-A and RSV-B infection were 151, 142, and 116 respectively. Systemic steroids were used in $39.6 \%$ of patients.

Totally, LOS of patients using steroid was longer than steroid free patients.

In RSV-A and RSV-B, LOSs in patients without use of steroid were shorter than patients with steroid treatment $(4.2 \pm 1.1$ vs $5.9 \pm 2.9, p<0.01,4.3 \pm 1.7$ vs $6.3 \pm 4.3 p<0.01$ respectively)

Also in rhinoviral infectious cases, LOSs in patients without use of steroid were shorter than patients with steroid treatment $(4.0 \pm 1.9$ vs $4.6 \pm 1.8, \mathrm{p}=0.02$ ).

But, there was not significant difference between steroid use patients and no use patients $(4.0 \pm 2.3$ vs $4.3 \pm 1.4, p=0.39)$ in group of rhinovirus infected patients over 12 months old.

\section{Conclusions}

In patient of bronchiolitis showing wheezing in young children, there is not evidence to support use of steroid in bronchiolitis children. The use of steroid in bronchiolitis might make the illness worse. But the use of steroid in older children infected by rhinovirus could be considered. Further study with multicenter randomized controlled trial (RCT) that assesses the clinical effectiveness of corticosteroids treatment for bronchiolitis considering the causative pathogens is needed.

\section{Limitations in this study}

-Retrospective

-No analysis using clinical score and allergy history 